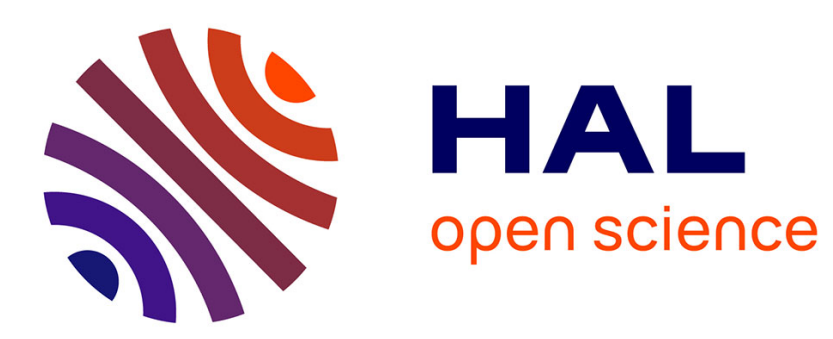

\title{
On an analytical Self-Consistent model for internal stress prediction in fiber-reinforced composites submitted to hygroelastic load
}

\author{
Sylvain Fréour, Frédéric Jacquemin, Ronald Guillen
}

\section{- To cite this version:}

Sylvain Fréour, Frédéric Jacquemin, Ronald Guillen. On an analytical Self-Consistent model for internal stress prediction in fiber-reinforced composites submitted to hygroelastic load. Journal of Reinforced Plastics and Composites, 2005, 24, pp.1365-1377. 10.1177/0731684405049887 . hal-01007141

\author{
HAL Id: hal-01007141 \\ https://hal.science/hal-01007141
}

Submitted on 16 Oct 2017

HAL is a multi-disciplinary open access archive for the deposit and dissemination of scientific research documents, whether they are published or not. The documents may come from teaching and research institutions in France or abroad, or from public or private research centers.
L'archive ouverte pluridisciplinaire HAL, est destinée au dépôt et à la diffusion de documents scientifiques de niveau recherche, publiés ou non, émanant des établissements d'enseignement et de recherche français ou étrangers, des laboratoires publics ou privés. 


\title{
On an Analytical Self-consistent Model for Internal Stress Prediction in Fiber-reinforced Composites Submitted to Hygroelastic Load
}

\author{
S. FRÉOUR, F. JACQUEMIN* AND R. GUILLÉN \\ Institut de Recherche en Génie Civil et Mécanique (UMR CNRS 6183) \\ IUT de Saint-Nazaire, Boulevard de l'Université \\ BP 406, 44602 Saint-Nazaire, Cedex, France
}

The aim of this work is to demonstrate a fully explicit analytical micromechanical self consistent approach dedicated to mechanical states prediction in both the fiber and the matrix of composite structures submitted to a transient hygroelastic load. The analytical forms obtained are applied to the case of carbon epoxy composites. Rigorous continuum mechanics formalisms are used for the determination of the required time and space dependent macroscopic stresses. The reliability of the new approach is checked through a comparison between the local stress states calculated in both the resin and the fiber according to the new closed form solutions and the equivalent numerical model.

KEY WORDS: self consistent model, analytical approach, hygroelastic stresses.

\section{INTRODUCTION}

$\mathbf{F}$ OR ABOUT TWENTY years, composite materials have become an alternative solution for structure elements in application fields requiring a very high strength-to-weight ratio. In aerospace engineering, for instance, composite laminates can be substituted for advanced metallic alloys (titanium alloys) or metal matrix composites (Al-SiC). However, designing composite components for such applications requires taking care of their durability in hygroscopic environments. Contrary to metallic material or metal matrix composites, carbon/epoxy laminates do actually absorb water, when placed in humid surroundings. Since carbon fibers do not absorb water, the moisture content of the constituents of a laminate (both the fibers and the resin) exhibits strong heterogeneities. Furthermore, the coefficients of moisture expansion (CME) of epoxy resins and carbon fibers are also strongly heterogeneous. Consequently, any hygroscopic load applied on a composite structure induces internal stresses and strains. Thus, a better understanding of the distribution of these local mechanical states is necessary to predict a possible damage occurrence in the material during its manufacturing process or service life.

*Author to whom correspondence should be addressed. E-mail: jacquemin @lamm.univ-nantes.fr 
In a previous work [1], a numerical self-consistent (SC) micromechanical model has been combined to continuum mechanics formalisms in order to predict the mechanical states at each scale of carbon-epoxy composite pipes submitted to transient hygroscopic fields. The macroscopic stresses and strains in each ply of the structure were deduced as a function of time and space using continuum mechanics formalisms. The SC framework was dedicated to the determination of the average pseudomacroscopic mechanical states in the components of each ply: the epoxy matrix on one hand and the carbon fiber on the other. Convenient closed-form solutions were previously developed in order to enable an easier programming and a faster computation of the macroscopic fields [2]. However, only numerical approaches were used for the determination of the pseudomacroscopic mechanical states.

In the present work, a suitable analytical SC model ensuring the calculation of the pseudomacroscopic stresses and strains is rigorously demonstrated. The model is established using a realistic assumption compatible with the microstructure of composite materials. Particular care is taken to express satisfactory closed-form solutions for every component of Morris' tensor that is especially involved in the hygroelastic morphologic effects. Finally, the new forms obtained are compared to the previous numerical SC framework, in order to verify the validity of the present proposal.

\section{ANALYTICAL FORMS FOR MORRIS' TENSOR FOR FIBER MORPHOLOGY}

The classical SC framework corresponding to the formalism introduced by Kröner [3] and Eshelby [4] is based on the mechanical treatment of the interactions between ellipsoidal heterogeneous inclusions embedded in an infinite medium. The average macroscopic elastic properties $\mathbf{L}^{I}$ of the composite are related to the morphology assumed for elementary inclusions, through Morris' tensor $\mathbf{E}^{I}$. Spherical inclusions only were initially considered by Morris [5]. In the case, when ellipsoidal shaped inclusions have to be taken into account, the following general form enables the calculation of the components of this tensor (see the works of Asaro and Barnett [6] or Kocks et al. [7]):

$$
\left\{\begin{array}{l}
E_{i j k l}^{I}=\frac{1}{4 \pi} \int_{0}^{\pi} \sin \theta d \theta \int_{0}^{2 \pi} \gamma_{i k j l} d \varphi \\
\gamma_{i k j l}=K_{i k}^{1}(\xi) \xi_{j} \xi_{l}
\end{array}\right.
$$

In the case of an orthotropic macroscopic symmetry, the components $K_{j p}(\xi)$ were given by Kröner [8]:

$$
\mathbf{K}=\left[\begin{array}{ccc}
L_{11}^{I} \xi_{1}^{2}+L_{66}^{I} \xi_{2}^{2}+L_{55}^{I} \xi_{3}^{2} & \left(L_{12}^{I}+L_{66}^{I}\right) \xi_{1} \xi_{2} & \left(L_{13}^{I}+L_{55}^{I}\right) \xi_{1} \xi_{2} \\
\left(L_{12}^{I}+L_{66}^{I}\right) \xi_{1} \xi_{2} & L_{66}^{I} \xi_{1}^{2}+L_{22}^{I} \xi_{2}^{2}+L_{44}^{I} \xi_{3}^{2} & \left(L_{23}^{I}+L_{44}^{I}\right) \xi_{2} \xi_{3} \\
\left(L_{13}^{I}+L_{55}^{I}\right) \xi_{1} \xi_{2} & \left(L_{23}^{I}+L_{44}^{I}\right) \xi_{2} \xi_{3} & L_{55}^{I} \xi_{1}^{2}+L_{44}^{I} \xi_{2}^{2}+L_{33}^{I} \xi_{3}^{2}
\end{array}\right]
$$

with

$$
\xi_{1}=\frac{\sin \theta \cos \varphi}{a_{1}}, \quad \xi_{2}=\frac{\sin \theta \sin \varphi}{a_{2}}, \quad \xi_{3}=\frac{\cos \theta}{a_{3}}
$$


where $2 a_{1}, 2 a_{2}, 2 a_{3}$ are the lengths of the principal axes of the ellipsoid, assumed to be respectively parallel to the longitudinal, transverse, and normal directions of the sample reference frame.

Some analytical forms for Morris' tensor are available in the literature; the interested reader can, for instance, refer to the books of Mura [9], Kocks et al. [7], or Qiu and Weng [10]. Nevertheless, these forms were established considering either spherical, disc-shaped or fiber-shaped inclusions embedded in an ideally isotropic macroscopic medium, that is incompatible with the strong elastic anisotropy exhibited by fiber-reinforced composites at a macroscopic scale [11].

In the case of carbon-epoxy composites, a transversely isotropic macroscopic behavior being coherent with fiber shape is actually expected (and predicted by the numerical computations). Assuming that the longitudinal (subscripted 1) axis is parallel to the fiber axis, one obtains the following conditions for the semilengths of the microstructure representative ellipsoid: $a_{1} \rightarrow \infty, a_{2}=a_{3}$. Moreover, the macroscopic elastic stiffness should satisfy:

$$
\mathbf{L}^{I}=\left[\begin{array}{cccccc}
L_{11}^{I} & L_{12}^{I} & L_{12}^{I} & 0 & 0 & 0 \\
L_{12}^{I} & L_{22}^{I} & L_{23}^{I} & 0 & 0 & 0 \\
L_{12}^{I} & L_{23}^{I} & L_{22}^{I} & 0 & 0 & 0 \\
0 & 0 & 0 & L_{44}^{I} & 0 & 0 \\
0 & 0 & 0 & 0 & L_{55}^{I} & 0 \\
0 & 0 & 0 & 0 & 0 & L_{55}^{I}
\end{array}\right]
$$

Putting (4) in (2) and (3) and considering the conditions $a_{2}=a_{3}, a_{1} \rightarrow \infty$ (that can be rewritten as $\xi_{1} \ll \xi_{2}, \xi_{3}$ ) leads to the following simplifications of $\mathbf{K}$ tensor:

$$
\mathbf{K}=\left[\begin{array}{ccc}
L_{55}^{I}\left(\xi_{2}^{2}+\xi_{3}^{2}\right) & \left(L_{12}^{I}+L_{55}^{I}\right) \xi_{1} \xi_{2} & \left(L_{12}^{I}+L_{55}^{I}\right) \xi_{1} \xi_{2} \\
\left(L_{12}^{I}+L_{55}^{I}\right) \xi_{1} \xi_{2} & L_{22}^{I} \xi_{2}^{2}+L_{44}^{I} \xi_{3}^{2} & \left(L_{23}^{I}+L_{44}^{I}\right) \xi_{2} \xi_{3} \\
\left(L_{12}^{I}+L_{55}^{I}\right) \xi_{1} \xi_{2} & \left(L_{23}^{I}+L_{44}^{I}\right) \xi_{2} \xi_{3} & L_{44}^{I} \xi_{2}^{2}+L_{22}^{I} \xi_{3}^{2}
\end{array}\right]
$$

The determination of Morris' tensor requires the determination of the inverse of $\mathbf{K}$ tensor that is involved in the calculation of $\gamma$.

Due to the conditions listed above over the dimensions $a_{1}, a_{2}$, and $a_{3}$ of the considered fiber-shaped inclusions, and according to (5), the inequation $K_{i i} \gg K_{i j}$ (with $i \neq j$ ) has also to be considered in order to find the most significant terms (the others being negligible) of $\mathbf{K}^{-1}$.

$$
\mathbf{K}^{1}=\left[\begin{array}{ccc}
H_{11} & 0 & 0 \\
0 & H_{22} & H_{23} \\
0 & H_{23} & H_{22}
\end{array}\right]+\mathbf{e}
$$


where

$$
\left\{\begin{array}{l}
\mathbf{e} \rightarrow 0 \\
H_{11}=\frac{a_{2}^{2}}{L_{55}^{I}\left(k_{2}^{2}+k_{3}^{2}\right)} \\
H_{22}=\frac{a_{2}^{2}\left[L_{22}^{I}\left(k_{2}^{2}+2 k_{3}^{2}\right)-L_{23}^{I} k_{2}^{2}\right]}{L_{22}^{I}\left(L_{22}^{I}-L_{23}^{I}\right)\left(k_{2}^{2}+k_{3}^{2}\right)^{2}} \\
H_{23}=-\frac{a_{2}^{2} k_{2} k_{3}\left(L_{22}^{I}+L_{23}^{I}\right)}{L_{22}^{I}\left(L_{22}^{I}-L_{23}^{I}\right)\left(k_{2}^{2}+k_{3}^{2}\right)^{2}} \\
\text { with } k_{1}=\sin \theta \cos \phi, k_{2}=\sin \theta \sin \phi, \text { and } k_{3}=\cos \theta
\end{array}\right.
$$

It is to be noted that the highest $a_{1}$ power factors implied in (7) is 0 , due to the inversion of $\mathbf{K}$. Any other factor involves negative powers of $a_{1}$ that are negligible compared to the terms given in Equations (7).

Now, it is obvious, that (1)-(3), (6) and (7) lead to drastic simplifications of Morris' tensor (1), in the case of fibers. Actually, one obtains (in contracted notation i.e., $E_{i j}^{I}$ components are given here):

$$
\mathbf{E}^{I}=\left[\begin{array}{cccccc}
0 & 0 & 0 & 0 & 0 & 0 \\
0 & \frac{3}{8 L_{22}^{\mathrm{I}}}+\frac{1}{4 L_{22}^{\mathrm{I}}-4 L_{23}^{\mathrm{I}}} & \frac{L_{22}^{\mathrm{I}}+L_{23}^{\mathrm{I}}}{8 L_{22}^{\mathrm{I}} L_{23}^{\mathrm{I}}-8 L_{22}^{\mathrm{I}^{2}}} & 0 & 0 & 0 \\
0 & \frac{L_{22}^{\mathrm{I}}+L_{23}^{\mathrm{I}}}{8 L_{22}^{\mathrm{I}} L_{23}^{\mathrm{I}}-8 L_{22}^{\mathrm{I}^{2}}} & \frac{3}{8 L_{22}^{\mathrm{I}}}+\frac{1}{4 L_{22}^{\mathrm{I}}-4 L_{23}^{\mathrm{I}}} & 0 & 0 & 0 \\
0 & 0 & 0 & \frac{1}{8 L_{22}^{\mathrm{I}}}+\frac{1}{4 L_{22}^{\mathrm{I}}-4 L_{23}^{\mathrm{I}}} & 0 & 0 \\
0 & 0 & 0 & 0 & \frac{1}{8 L_{55}^{\mathrm{I}}} & 0 \\
0 & 0 & 0 & 0 & 0 & \frac{1}{8 L_{55}^{\mathrm{I}}}
\end{array}\right]
$$

\section{CHECKING OF NEW ANALYTICAL FORMS FOR MORRIS' TENSOR}

The new closed-form (8) demonstrated for the components of Morris' tensor assuming the proper fiber morphology expected in composite materials have to be compared with the classical numerical calculations based on (1)-(3), in order to assess their validity.

The case of T300/5208 laminate containing a fiber volume fraction of $60 \%$ was considered. The behavior of each composite is governed by its constituents, i.e., the properties of the fibers, the surrounding matrix, and the relative amount of the fibers and matrix in the material. Calculations were performed assuming the initial local elastic properties listed in Table 1 ( $Y, v$, and $G$ stands respectively for Young's modulus, Poisson's ratio, and Coulomb's modulus).

The macroscopic stiffness $\mathbf{L}^{I}$ obtained with the fully numerical elastic SC model is summarized in Table 2. It was determined assuming the following values for the length of 
Table 1. Mechanical properties of T300/5208 constituents.

\begin{tabular}{lccccc}
\hline & $\boldsymbol{Y}_{\mathbf{1}}$ (GPa) & $\boldsymbol{Y}_{\mathbf{2}}, \boldsymbol{Y}_{\mathbf{3}}$ (GPa) & $v_{\mathbf{1 2}}, v_{\mathbf{1 3}}$ & $\mathbf{G}_{\mathbf{2 3}}$ (GPa) & $\mathbf{G}_{\mathbf{1 2}}$ (GPa) \\
\hline T300 fibers [12] & 230 & 15 & 0.2 & 7 & 15 \\
N5208 epoxy matrix [13] & 4.5 & 4.5 & 0.4 & 1.6 & 1.6 \\
\hline
\end{tabular}

Table 2. Macroscopic elastic constants of a T300/5208 composite, according to the fully numerical SC elastic model.

\begin{tabular}{lcccc}
\hline$Y_{1}(\mathrm{GPa})$ & $Y_{2}, Y_{3}(\mathrm{GPa})$ & $v_{12}, v_{13}$ & $G_{23}(\mathrm{GPa})$ & $G_{12}(\mathrm{GPa})$ \\
\hline 139.6 & 9.8 & 0.28 & 3.5 & 6.4 \\
\hline
\end{tabular}

Table 3. Comparison between the numerical and analytical estimations for Morris' tensor components.

\begin{tabular}{lcccccc}
\hline Approach & $\begin{array}{c}E_{11}^{\prime} \\
\left(\mathrm{TPa}^{1}\right)\end{array}$ & $\begin{array}{c}E_{12}^{\prime} \\
\left(\mathrm{TPa}^{1}\right)\end{array}$ & $\begin{array}{c}E_{22}^{\prime} \\
\left(\mathrm{TPa}^{1}\right)\end{array}$ & $\begin{array}{c}E_{23}^{\prime} \\
\left(\mathrm{TPa}^{1}\right)\end{array}$ & $\begin{array}{c}E_{44}^{\prime} \\
\left(\mathrm{TPa}^{1}\right)\end{array}$ & $\begin{array}{c}E_{55}^{\prime} \\
\left(\mathrm{TPa}^{1}\right)\end{array}$ \\
\hline Numerical & 0.03 & 0.01 & 60.8 & 24.7 & 50.2 & 17.9 \\
Analytical & 0.00 & 0.00 & 64.1 & 28.2 & 46.1 & 17.9 \\
\hline
\end{tabular}

the semiaxis of the inclusions, in order to take into account the proper fiber microstructure of the material: $a_{2}=a_{3}=1$, and $a_{1}=100$. The implicit relation used to proceed to the calculation of $\mathbf{L}^{I}$ is very classical. The interested reader can refer to [14], where an extensive demonstration is given and leads to:

$$
\mathbf{L}^{I}=\left\langle\left(\mathbf{L}^{\alpha}+\mathbf{L}^{I}: \mathbf{R}^{I}\right)^{1}:\left(\mathbf{L}^{I}+\mathbf{L}^{I}: \mathbf{R}^{I}\right): \mathbf{L}^{\alpha}\right\rangle_{\alpha=f, m}
$$

where $\mathbf{L}^{\alpha}$ is the elastic stiffness of the studied component, i.e., the fiber or the matrix (that will respectively be denoted in the following by the superscripts $f$ and $m$ ). The brackets \langle\rangle stand for volume averages. For instance, $\left\langle\mathbf{A}^{\alpha}\right\rangle_{\alpha=f, m}=v^{f} \mathbf{A}^{f}+v^{m} \mathbf{A}^{m}$, with $v^{f}$ and $v^{m}$ the respective volume fractions of the fiber and the matrix in the ply. $\mathbf{R}^{I}$ represents the so-called reaction tensor that expresses the elastic interactions due to the morphology assumed for the elementary constituents of the composite material. It satisfies:

$$
\mathbf{R}^{I}=\left(\mathbf{L}^{I^{1}}-\mathbf{E}^{I}\right): \mathbf{E}^{I^{1}}
$$

The corresponding Morris' tensors calculated using either the classical framework (1)-(3) or the analytical forms (8) are given in Table 3. The results obtained in Table 3 show a good agreement between the fully analytical calculations of Morris' tensor and the classical numerical integration. Thus, the closed-form solutions demonstrated in the section on "Analytical forms for Morris tensor for fiber morphology" are satisfying to describe the elastic interactions induced by the specific microstructure of composites reinforced by carbon fibers.

Next, the simplified form obtained for Morris' tensor is taken into account in order to demonstrate fully analytical relations for the internal stresses and strains in both the fibers and the matrix of carbon-epoxy composites submitted to a macroscopic hygroelastic load. 


\section{NUMERICAL AND ANALYTICAL PREDICTIONS OF THE PSEUDOMACROSCOPIC MECHANICAL STATES WITH SC MODEL}

\section{Numerical SC model Extended to a Hygroelastic Load}

Within Kröner [3] and Eshelby [4] self-consistent framework, the hygrothermal dilatation generated by a moisture content increment $\Delta C^{\alpha}$ is treated as a transformation strain exactly like the thermal dilatation occurring after a temperature increment (that last case was extensively discussed in the literature, see for example Kocks et al. [7]). Thus, the pseudomacroscopic stresses $\boldsymbol{\sigma}^{\alpha}$ in the considered constituent are given by:

$$
\boldsymbol{\sigma}^{\alpha}=\mathbf{L}^{\alpha}:\left(\boldsymbol{\varepsilon}^{\alpha}-\boldsymbol{\beta}^{\alpha} \Delta \mathbf{C}^{\alpha}\right)
$$

Here, $\boldsymbol{\beta}$ stands for the coefficients of moisture expansion (CME). $\boldsymbol{\varepsilon}$ is the strain tensor and $\Delta C$ the moisture content. Generally, it differs at the macroscopic and pseudomacroscopic scales. Actually, carbon fibers usually do not absorb moisture. As a consequence, the mass of water contained by the composite is either found in the matrix, locally trapped in porosities (Mensitieri et al. [15]) or located where fiber debonding occurs.

Replacing the superscripts $\alpha$ by $I$ in (11) leads to the stress strain relation that holds at a macroscopic scale.

$$
\boldsymbol{\sigma}^{I}=\mathbf{L}^{I}:\left(\varepsilon^{I}-\boldsymbol{\beta}^{I} \Delta C^{I}\right)
$$

The so-called 'scale-transition relation' enabling to determine the local stresses and strains from the macroscopic mechanical states was demonstrated by Eshelby [4] in a fundamental work, starting from the assumption that the elementary inclusions (here the matrix and the fiber) have ellipsoidal shapes:

$$
\boldsymbol{\sigma}^{\alpha}-\boldsymbol{\sigma}^{I}=-\mathbf{L}^{I}: \mathbf{R}^{I}:\left(\boldsymbol{\varepsilon}^{\alpha}-\boldsymbol{\varepsilon}^{I}\right)
$$

Actually, (13) is not very useful, because both the unknown pseudomacroscopic stresses and the strains appear. Nevertheless, combining (11)-(13) enables to find the following expression for the pseudomacroscopic strain (the demonstration is available in [1]):

$$
\boldsymbol{\varepsilon}^{\alpha}=\left(\mathbf{L}^{\alpha}+\mathbf{L}^{I}: \mathbf{R}^{I}\right)^{1}:\left[\left(\mathbf{L}^{I}+\mathbf{L}^{I}: \mathbf{R}^{I}\right): \varepsilon^{I}+\mathbf{L}^{\alpha}: \boldsymbol{\beta}^{\alpha} \Delta C^{\alpha}-\mathbf{L}^{I}: \boldsymbol{\beta}^{\mathrm{I}} \Delta C^{I}\right]
$$

Moreover, it was established by Hill in 1967 [16], that the self-consistent model was compatible with the following volume averages on both the pseudomacroscopic stresses and the strains:

$$
\begin{aligned}
& \left\langle\boldsymbol{\sigma}^{\alpha}\right\rangle_{\alpha=f, m}=\boldsymbol{\sigma}^{I} \\
& \left\langle\boldsymbol{\varepsilon}^{\alpha}\right\rangle_{\alpha=f, m}=\boldsymbol{\varepsilon}^{I}
\end{aligned}
$$

It was demonstrated by Jacquemin et al. in [1] that the macroscopic CME should satisfy:

$$
\boldsymbol{\beta}^{I}=\frac{\Delta C^{\alpha}}{\Delta C^{I}} \mathbf{L}^{I^{1}}\left\langle\left(\mathbf{L}^{\alpha}+\mathbf{L}^{I}: \mathbf{R}^{I}\right)^{1}\right\rangle_{\alpha f, m}^{1}:\left\langle\left(\mathbf{L}^{\alpha}+\mathbf{L}^{I}: \mathbf{R}^{I}\right)^{1}: \mathbf{L}^{\alpha}: \beta^{\alpha}\right\rangle_{\alpha f, m}
$$

For a given applied macroscopic hygroelastic load $\left\{\boldsymbol{\sigma}^{I}, \Delta C^{I}\right\}$, one can easily determine $\boldsymbol{\varepsilon}^{I}$ through (12), provided that the effective elastic behavior $\mathbf{L}^{I}$ of the ply has been calculated 
using (9), whereas the macroscopic CME are given by (16). Then, the pseudo-macroscopic strains are determined through (14).

\section{Analytical Solution for the Pseudomacroscopic Mechanical States}

In fact, the epoxy matrix is usually isotropic, so that three components only have to be considered for its elastic constants: $L_{11}^{m}, L_{12}^{m}$, and $L_{44}^{m}$. One moisture expansion coefficient is sufficient to describe the hygroscopic behavior of the matrix: $\beta_{11}^{m}$. In the case of the carbon fibers, a transverse isotropy is generally observed. Thus, the corresponding elasticity constants depend on the following components: $L_{11}^{f}, L_{12}^{f}, L_{22}^{f}, L_{23}^{f}, L_{44}^{f}$, and $L_{55}^{f}$. Moreover, since the carbon fiber does not absorb water, its CME $\beta_{11}^{f}$ and $\beta_{22}^{f}$ will not be involved in the mechanical states determination. Introducing these additional assumptions in (14), and taking into account the form (8) obtained for Morris' tensor, one can deduce the following strain tensors for both the matrix and the fibers:

$$
\boldsymbol{\varepsilon}^{\alpha}=\left[\begin{array}{lll}
\varepsilon_{11}^{\alpha} & \varepsilon_{12}^{\alpha} & \varepsilon_{13}^{\alpha} \\
\varepsilon_{12}^{\alpha} & \varepsilon_{22}^{\alpha} & \varepsilon_{23}^{\alpha} \\
\varepsilon_{13}^{\alpha} & \varepsilon_{23}^{\alpha} & \varepsilon_{33}^{\alpha}
\end{array}\right]
$$

where, in the case of the matrix,

$$
\left\{\begin{array}{l}
\varepsilon_{11}^{m}=\varepsilon_{11}^{I} \\
\varepsilon_{12}^{m}=\frac{2 L_{55}^{I} \varepsilon_{12}^{I}}{L_{55}^{I}+L_{44}^{m}} \\
\varepsilon_{13}^{m}=\frac{2 L_{55}^{I} \varepsilon_{13}^{I}}{L_{55}^{I}+L_{44}^{m}} \\
\varepsilon_{22}^{m}=\frac{N_{1}^{m}+N_{2}^{m}+N_{3}^{m}+N_{4}^{m}}{D_{1}^{m}} \\
\varepsilon_{23}^{m}=\frac{2 L_{22}^{I}\left(L_{22}^{I}-L_{23}^{I}\right) \varepsilon_{23}^{I}}{2 L_{22}^{I^{2}}+L_{23}^{I}\left(L_{44}^{I}-L_{44}^{m}\right)+L_{22}^{I}\left(3 L_{44}^{m}-2 L_{23}^{I}-3 L_{44}^{I}\right)} \\
\varepsilon_{33}^{m}=\varepsilon_{22}^{m}-4 L_{22}^{I} \frac{\left(L_{22}^{I}-L_{23}^{I}\right)\left(\varepsilon_{22}^{I}-\varepsilon_{33}^{I}\right)}{L_{22}^{I^{2}}+3 L_{22}^{I}\left(L_{11}^{m}-L_{12}^{m}\right)-L_{23}^{I}\left(L_{11}^{m}+L_{23}^{I}-L_{12}^{m}\right)} \\
N_{1}^{m}=\beta_{11}^{m}\left(L_{11}^{m}+2 L_{12}^{m}\right) \Delta C^{m}-\left(\beta_{11}^{I} L_{12}^{I}+\beta_{22}^{I}\left(L_{22}^{I}+L_{23}^{I}\right)\right) \Delta C^{I} \\
N_{2}^{m}=\left(L_{12}^{I}-L_{12}^{m}\right) \varepsilon_{11}^{I} \\
N_{3}^{m}=\frac{L_{22}^{I}\left\{L_{22}^{I}\left(5 L_{11}^{m}-L_{12}^{m}+3 L_{22}^{I}\right)-L_{23}^{I}\left(3 L_{11}^{m}+L_{12}^{m}+4 L_{22}^{I}\right)+L_{23}^{I^{2}}\right\}}{\left(3 L_{22}^{I}-L_{23}^{I}\right)\left(L_{11}^{m}-L_{12}^{m}\right)+L_{22}^{I^{2}}-L_{23}^{I^{2}}} \varepsilon_{22}^{I} \\
N_{4}^{m}=\frac{L_{22}^{I}\left\{L_{22}^{I}\left(L_{11}^{m}-5 L_{12}^{m}-L_{22}^{I}\right)+L_{23}^{I}\left(L_{11}^{m}+3 L_{12}^{m}+4 L_{22}^{I}\right)-3 L_{23}^{I^{2}}\right\}}{\left(3 L_{22}^{I}-L_{23}^{I}\right)\left(L_{11}^{m}-L_{12}^{m}\right)+L_{22}^{I^{2}}-L_{23}^{I^{2}}} \varepsilon_{33}^{I} \\
D_{1}^{m}=L_{11}^{m}+L_{12}^{m}+L_{22}^{I}-L_{23}^{I}
\end{array}\right.
$$


The pseudomacroscopic stress tensors are deduced from the strains using (11). Thus, in the matrix, one will have:

$$
\sigma^{m}=\left[\begin{array}{ccc}
\sigma_{11}^{m} & 2 L_{44}^{m} \varepsilon_{12}^{m} & 2 L_{44}^{m} \varepsilon_{13}^{m} \\
2 L_{44}^{m} \varepsilon_{12}^{m} & \sigma_{22}^{m} & 2 L_{44}^{m} \varepsilon_{23}^{m} \\
2 L_{44}^{m} \varepsilon_{13}^{m} & 2 L_{44}^{m} \varepsilon_{23}^{m} & \sigma_{33}^{m}
\end{array}\right]
$$

with

$$
\left\{\begin{array}{l}
\sigma_{11}^{m}=L_{11}^{m} \varepsilon_{11}^{m}+L_{12}^{m}\left(\varepsilon_{22}^{m}+\varepsilon_{33}^{m}\right)-\beta_{11}^{m}\left(L_{11}^{m}+2 L_{12}^{m}\right) \Delta C^{m} \\
\sigma_{22}^{m}=L_{11}^{m} \varepsilon_{22}^{m}+L_{12}^{m}\left(\varepsilon_{11}^{m}+\varepsilon_{33}^{m}\right)-\beta_{11}^{m}\left(L_{11}^{m}+2 L_{12}^{m}\right) \Delta C^{m} \\
\sigma_{33}^{m}=L_{11}^{m} \varepsilon_{33}^{m}+L_{12}^{m}\left(\varepsilon_{11}^{m}+\varepsilon_{22}^{m}\right)-\beta_{11}^{m}\left(L_{11}^{m}+2 L_{12}^{m}\right) \Delta C^{m}
\end{array}\right.
$$

The local mechanical states in the fiber are provided by Hill's strains and stresses average laws (15):

$$
\begin{gathered}
\boldsymbol{\varepsilon}^{f}=\frac{1}{v^{f}} \boldsymbol{\varepsilon}^{I}-\frac{v^{m}}{v^{f}} \boldsymbol{\varepsilon}^{m} \\
\boldsymbol{\sigma}^{f}=\frac{1}{v^{f}} \boldsymbol{\sigma}^{I}-\frac{v^{m}}{v^{f}} \boldsymbol{\sigma}^{m}
\end{gathered}
$$

In order to check the validity of these closed-form solutions (18) and (19), one can perform a simple test. Considering a pure matrix, the following transformation rules have to hold:

$$
\begin{aligned}
\Delta C^{m} & =\Delta C^{I}, \quad L_{11}^{I}=L_{22}^{I}=L_{11}^{m}, \quad L_{12}^{I}=L_{23}^{I}=L_{12}^{m}, \\
L_{44}^{I} & =L_{55}^{I}=L_{44}^{m}, \quad \beta_{11}^{I}=\beta_{22}^{I}=\beta_{11}^{m}
\end{aligned}
$$

Introducing (24) in (18) and (19), one gets the following simplifications:

$$
\left\{\begin{array}{l}
N_{1}^{m}=\beta_{11}^{m}\left(L_{11}^{m}+2 L_{12}^{m}\right) \Delta C^{m}-\left(\beta_{11}^{m} L_{12}^{m}+\beta_{11}^{m}\left(L_{11}^{m}+L_{12}^{m}\right)\right) \Delta C^{m}=0 \\
N_{2}^{m}=N_{2}^{m}=\left(L_{12}^{I}-L_{12}^{m}\right) \varepsilon_{11}^{I}=0 \\
N_{3}^{m}=\frac{L_{11}^{m}\left\{L_{11}^{m}\left(8 L_{11}^{m}-L_{12}^{m}\right)-L_{12}^{m}\left(7 L_{11}^{m}+L_{12}^{m}\right)+L_{12}^{m^{2}}\right\}}{\left(3 L_{11}^{m}-L_{12}^{m}\right)\left(L_{11}^{m}-L_{12}^{m}\right)+L_{11}^{m^{2}}-L_{12}^{m^{2}}} \varepsilon_{22}^{I}=L_{11}^{m} \frac{8 L_{11}^{m}\left(L_{11}^{m}-L_{12}^{m}\right)}{4 L_{11}^{m}\left(L_{11}^{m}-L_{12}^{m}\right)}=2 L_{11}^{m} \varepsilon_{22}^{I} \\
N_{4}^{m}=\frac{L_{11}^{m}\left\{-5 L_{11}^{m} L_{12}^{m}+L_{12}^{m}\left(3 L_{12}^{m}+5 L_{11}^{m}\right)-3 L_{12}^{m^{2}}\right\}}{\left(3 L_{22}^{I}-L_{23}^{I}\right)\left(L_{11}^{m}-L_{12}^{m}\right)+L_{22}^{I^{2}}-L_{23}^{I^{2}}} \varepsilon_{33}^{I}=0 \\
D_{1}^{m}=2 L_{11}^{m}
\end{array}\right.
$$




$$
\left\{\begin{array}{l}
\varepsilon_{11}^{m}=\varepsilon_{11}^{I} \\
\varepsilon_{12}^{m}=\frac{2 L_{44}^{m} \varepsilon_{12}^{I}}{L_{44}^{m}+L_{44}^{m}}=\varepsilon_{12}^{I} \\
\varepsilon_{13}^{m}=\frac{2 L_{44}^{m} \varepsilon_{13}^{I}}{L_{44}^{m}+L_{44}^{m}}=\varepsilon_{13}^{I} \\
\varepsilon_{22}^{m}=\frac{N_{1}^{m}+N_{2}^{m}+N_{3}^{m}+N_{4}^{m}}{D_{1}^{m}}=\varepsilon_{22}^{I} \\
\varepsilon_{23}^{m}=\frac{2 L_{11}^{m}\left(L_{11}^{m}-L_{12}^{m}\right) \varepsilon_{23}^{I}}{2 L_{11}^{m^{2}}+L_{12}^{m}\left(L_{44}^{m}-L_{44}^{m}\right)+L_{11}^{m}\left(3 L_{44}^{m}-2 L_{12}^{m}-3 L_{44}^{m}\right)}=\frac{2 L_{11}^{m}\left(L_{11}^{m}-L_{12}^{m}\right) \varepsilon_{23}^{I}}{2 L_{11}^{m^{2}}-2 L_{11}^{m} L_{12}^{m}}=\varepsilon_{23}^{I} \\
\varepsilon_{33}^{m}=\varepsilon_{22}^{m}-4 L_{11}^{m} \frac{\left(L_{11}^{m}-L_{12}^{m}\right)\left(\varepsilon_{22}^{I}-\varepsilon_{33}^{I}\right)}{L_{11}^{m^{2}}+3 L_{11}^{m}\left(L_{11}^{m}-L_{12}^{m}\right)-L_{12}^{m}\left(L_{11}^{m}+L_{12}^{m}-L_{12}^{m}\right)}=\varepsilon_{22}^{m}-\left(\varepsilon_{22}^{I}-\varepsilon_{33}^{I}\right)=\varepsilon_{33}^{I}
\end{array}\right.
$$

In the following section, the new analytical forms obtained for the internal pseudomacroscopic mechanical states are compared to the classical fully numerical model.

\section{COMPARISON BETWEEN ANALYTICAL AND NUMERICAL SC MODELS}

\section{Introduction}

Calculations were made for a T300/5208 carbon-epoxy composite. The closed-form formalism used in order to determine the mechanical stresses and strains in each ply of the structure is described by Jacquemin and Vautrin [2]. This model ensures the calculation of the macroscopic moisture content, too.

When the equilibrium state is reached, the maximum moisture content of the neat resin may be estimated from the maximum moisture content of the composite. By assuming that the fibers do not absorb any moisture, $\Delta C^{I}$ and $\Delta C^{m}$ are related by the expression given by Loos and Springer [17]:

$$
\Delta C^{I}=\Delta C^{m} W^{m}
$$

where $W^{m}$ is the weight fraction (percent) of the resin in the composite.

The preceding equation develops as follows:

$$
\frac{\Delta C^{m}}{\Delta C^{I}}=\frac{\rho^{I}}{v^{m} \rho^{m}}
$$

where $\rho^{I}$ and $\rho^{m}$ are respectively the composite and resin densities.

In the case of T300/5208, since the ratio between composite and resin densities is 1.33 , the maximum moisture content ratio given by (28) is about 3.33. Ply CME estimated through SC model (16), using Tables 2 and 4 as input parameters, are: $\beta_{11}^{I}=0.035$ and $\beta_{22}^{I}=1.026$. These values are compatible with the classical Tsai-Hahn model [11], usually used in order to achieve such estimation and based on rule of mixture assumptions, that leads to $\beta_{11}^{I}=0.026$ and $\beta_{22}^{I}=1.12$. 
Table 4. CME of the matrix N5208.

\begin{tabular}{lcc}
\hline & $\beta_{11}$ & $\beta_{\mathbf{2 2}}, \beta_{\mathbf{2 3}}$ \\
\hline N5208 epoxy matrix [13] & 0.6 & 0.6 \\
\hline
\end{tabular}
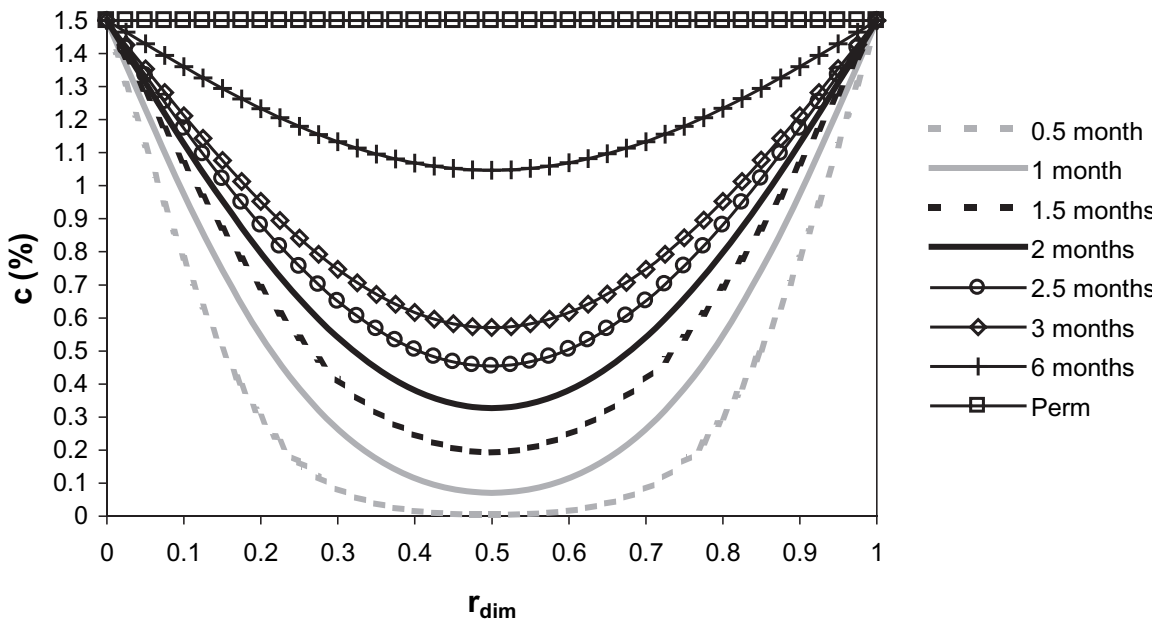

- 2.5 months

$\diamond-3$ months

+6 months

巴-Perm

Figure 1. Time dependent concentration profiles in $T 300 / 5208$ as a function of the normalized radial distance from the inner radius $r_{\text {dim }}$.

Thin laminated composite pipes, with $4 \mathrm{~mm}$ thickness, initially dry when exposed to an ambient fluid, made up of T300/5208 carbon-epoxy plies are considered for the determination of both macroscopic stresses and moisture content as a function of time and space.

\section{Results - Checking of the Analytical Model}

Figure 1 shows the time-dependent concentration profiles, resulting from the application of a boundary concentration $c_{0}$, as a function of the normalized radial distance from the inner radius $r_{\mathrm{dim}}$. At the beginning of the diffusion process, important concentration gradients occur near the external surfaces. The permanent concentration (noticed perm in the caption) holds with a constant value because of the symmetrical hygroscopic loading. The macroscopic mechanical states were calculated for two types of composite structures: (a) a unidirectionally reinforced cylinder, and (b) a $\left[55^{\circ} /-55^{\circ}\right]_{S}$ laminated cylinder.

Starting with the macroscopic stresses deduced from continuum mechanics, the local stresses in both the fiber and the matrix were calculated with either the new analytical forms or the fully numerical model. The comparison between the two approaches is plotted in Figures 2 and 3. These figures show a very good agreement between the numerical approach and the corresponding closed-form solutions. The slight differences appearing are due to the small deviations on the components of Morris' tensor calculated using the two approaches. Actually, it is not possible to assume the quasi-infinite length of the fiber along the longitudinal axis in the case of the numerical approach, because the 

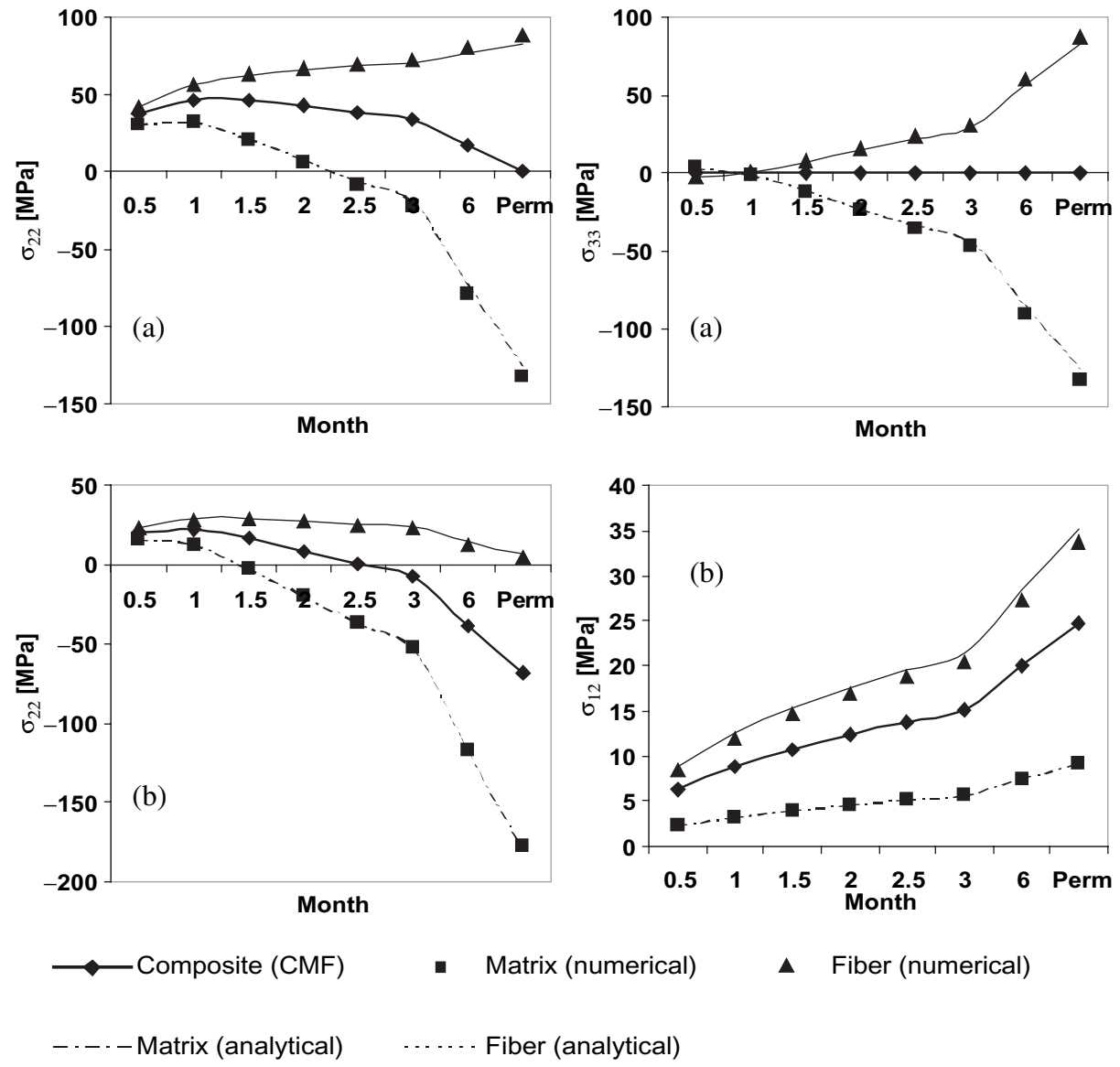

Figure 2. Local stresses in $T 300 / 5208$ composite for the central ply, in the case of (a) the unidirectionally reinforced composite and $(b)$ the $\left[+55^{\circ} 55^{\circ}\right]_{S}$ symmetric laminate. CMF stands for continuum mechanics formalisms.

numerical computation of Morris' tensor is highly time-consuming. Thus, the numerical SC model constitutes only an approximation of the real microstructure of the composite. In consequence, it seems that the new analytical forms, that are able to take into account the proper microstructure for the fibers, are not only more convenient, but also more reliable than the initially proposed numerical approach.

\section{Interpretation of the Simulations}

The highest level of macroscopic tensile stress is reached for the unidirectional composite, in the transverse direction and in the central ply of the structure (Figure 2). The transverse stresses probably exceed the macroscopic tensile strength in this direction. The choice of $\left[+55^{\circ} /-55^{\circ}\right]_{\mathrm{S}}$ laminate allows the reduction of macroscopic stress in the transverse direction. Nevertheless, a high shear stress rises along the time in the fibers of the central ply of such a structure (Figure 2).

Moreover, Figure 3 shows that the micromechanical model always predicts a very high compressive stress in the matrix of the inner ply whatever the laminate studied 

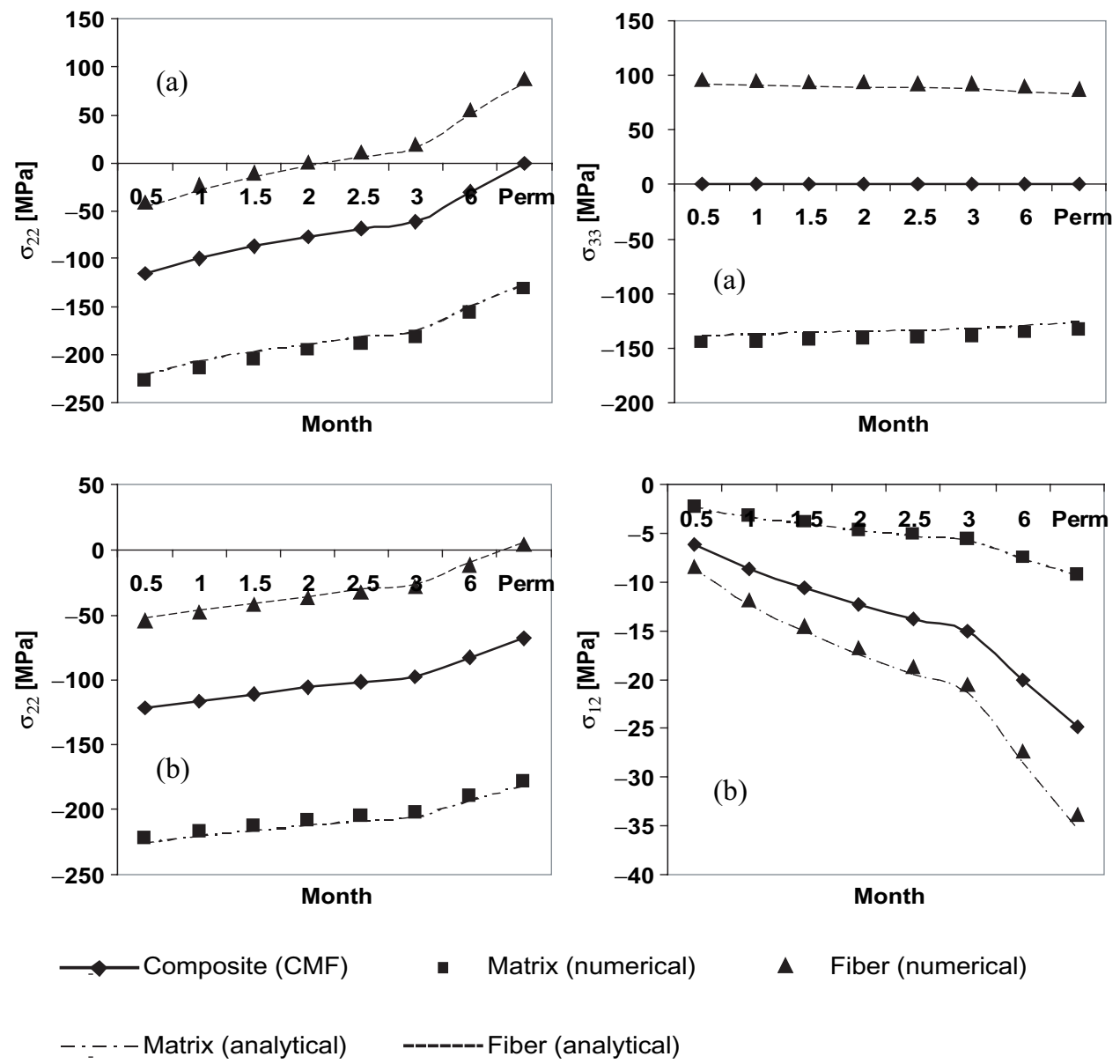

Figure 3. Local stresses in T300/5208 composite for the inner ply, in the case of (a) the unidirectionally reinforced composite and (b) the $\left[+55^{\circ} / 55^{\circ}\right]_{s}$ symmetric laminate. CMF stands for continuum mechanics formalisms.

(the macroscopic stress is negligible in the radial direction because thin structures are considered). These local stresses could help to explain damage occurrence in the surface of composite structures in fatigue.

This work demonstrates the complementarities of continuum mechanics and micromechanical models for the prediction of a possible damage in composite structures submitted to hygroelastic loads.

\section{CONCLUSIONS AND PERSPECTIVES}

In the present work, a rigorous fully analytical treatment of the classical Kröner and Eshelby self-consistent model including morphology effects was achieved. Especially, the determination of Morris' tensor was performed in a satisfactory agreement with the transverse macroscopic elastic anisotropy expected for the fiber shape that should be taken into account in order to satisfactorily represent the specific microstructure of carbon-fiber reinforced composites. 
The new closed-form solutions obtained for the components of Morris' tensor were introduced in the classical hygroelastic scale transition relation in order to analytically express the internal strains and stresses in both the fiber and the resin of a ply submitted to a hygroelastic load. The closed-form solution demonstrated in the present work was compared to the fully numerical self-consistent model for various geometrical arrangements of the fibers: unidirectional or laminated composites. A very good agreement is obtained between the two models for any component of the local stress tensors. It was also demonstrated that continuum mechanics and micromechanical models give complementary information about the occurrence of a possible damage during the loading of the structure.

Thus, the present analytical model, that works faster and is more convenient to program than the classical model, could be implemented in a calculation code combining both the continuum mechanics formalisms (necessary to determine the macroscopic stresses and strains in each ply) and the micromechanical model. This new software will constitute an accurate and powerful tool for the prediction of a possible damage in the material at every scale of a composite structure submitted to a transient hygroscopic stress. In further works, the effects of a cyclical hygrothermal load on both the local and the macroscopic mechanical states of carbon fiber-reinforced composites will be investigated.

\section{REFERENCES}

1. Jacquemin, F., Fréour, S. and Guillén, R. A Hygro elastic Self Consistent Model for Fiber Reinforced Composites, Journal of Reinforced Plastics and Composites, to be published.

2. Jacquemin, F. and Vautrin, A. (2002). A Closed form Solution for the Internal Stresses in Thick Composite Cylinders Induced by Cyclical Environmental Conditions, Composite Structures, 58: 19.

3. Kroner, E. (1958). Berechnung der elastischen Konstanten des Vielkristalls aus der Konstanten des Einkristalls, Zeitschrift für Physik, 151: 504518.

4. Eshelby, J. D. (1957). The Determination of the Elastic Field of an Ellipsoidal Inclusion, and Related Problems, Proc. Roy. Soc. London, A241: 376396.

5. Morris, P. R. (1970). Elastic Constants of Polycrystals, International Journal Engineering Science, 8: 4961.

6. Asaro, R. J. and Barnett, D. M. (1975). The Non uniform Transformation Strain Problem for an Anisotropic Ellipsoidal Inclusion, Journal of the Mechanics and Physics of Solids, 23: 7783.

7. Kocks, U. F., Tomé, C. N. and Wenk, H. R. (1998). Texture and Anisotropy, Cambridge University Press, Cambridge, UK.

8. Kroner, E. (1953). Dissertation, Technischen Hochschule Stuttgart.

9. Mura, T. (1982). Micromechanics of Defects in Solids, Martinus Nijhoff Publishers, The Hague, Netherlands.

10. Qiu, Y. P. and Weng, G. J. (1991). The Influence of Inclusion Shape on the Overall Elastoplastic Behavior of a Two phase Isotropic Composite, Int. J. Solids Structures, 27(12): 15371550.

11. Tsai, S. W. and Hahn, H. T. (1980). Introduction to Composite Materials, Technomic Publishing Co., Inc., Lancaster, Pennsylvania.

12. Soden, P. D., Hinton M. J. and Kaddour, A. S. (1998). Lamina Properties Lay up Configurations and Loading Conditions for a Range of Fiber reinforced Composite Laminates, Composites Science and Technology, 58: 10111022.

13. Tsai, S. W. (1987). Composite Design, 3rd edn, Think Composites.

14. Tomé, C. (1998). Tensor Properties of Textured Polycrystal, In: Texture and Anisotropy, Cambridge University Press, Cambridge, UK, pp. 283325.

15. Mensitieri, G. M., Del Nobile, M. A., Apicella, A. and Nicolais, L. (1995). Moisture matrix Interactions in Polymer Based Composite Materials, Revue de l'Institut Francais du Pétrole, 50: 551571.

16. Hill, R. (1967). The Essential Structure of Constitutive Laws for Metals Composites and Polycrystals, Journal of the Mechanics and Physics of Solids, 15: 7995.

17. Loos, A. C. and Springer, G. S. (1981). Moisture Absorption of Graphite Epoxy Composition Immersed in Liquids and in Humid Air, In: Environmental Effects on Composite Materials, Technomic Publishing, Westport, USA, pp. 3455. 